\title{
Dispersion Forces Stabilize Ice Coatings at Certain Gas Hydrate Interfaces That Prevent Water Wetting
}

\author{
Mathias Boström, ${ }^{*} \dagger, \ddagger \odot$ Robert W. Corkery, ${ }^{*},{ }^{\ddagger}$ Eduardo R. A. Lima, " Oleksandr I. Malyi,

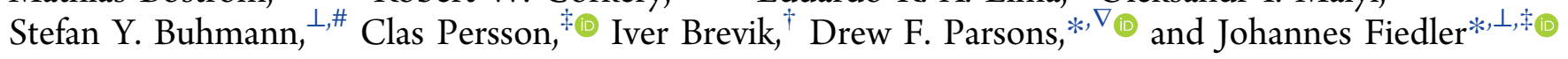 \\ ${ }^{\dagger}$ Department of Energy and Process Engineering, Norwegian University of Science and Technology, NO-7491 Trondheim, Norway \\ ${ }^{\ddagger}$ Centre for Materials Science and Nanotechnology, Department of Physics, University of Oslo, P.O. Box 1048 Blindern, NO-0316 \\ Oslo, Norway \\ OSurface and Corrosion Science, Department of Chemistry, KTH Royal Institute of Technology, SE 10044 Stockholm, Sweden \\ ${ }^{\S}$ Department of Applied Mathematics, Research School of Physics and Engineering, The Australian National University, Canberra \\ ACT 0200, Australia \\ "Programa de Pós-graduação em Engenharia Química, Universidade do Estado do Rio de Janeiro, CEP 20550-013, Rio de Janeiro, \\ RJ, Brazil \\ ${ }^{\perp}$ Physikalisches Institut, Albert-Ludwigs-Universität Freiburg, Hermann-Herder-Str. 3, 79104 Freiburg, Germany \\ ${ }^{\#}$ Freiburg Institute for Advanced Studies, Albert-Ludwigs-Universität Freiburg, Albertstr. 19, 79104 Freiburg, Germany \\ ${ }^{\nabla}$ School of Engineering and IT, Murdoch University, 90 South Street, Murdoch, WA 6150, Australia
}

ABSTRACT: Gas hydrates formed in oceans and permafrost occur in vast quantities on Earth representing both a massive potential fuel source and a large threat in climate forecasts. They have been predicted to be important on other bodies in our solar systems such as Enceladus, a moon of Saturn. $\mathrm{CO}_{2}^{-}$ hydrates likely drive the massive gas-rich water plumes seen and sampled by the spacecraft Cassini, and the source of these hydrates is thought to be due to buoyant gas hydrate particles. Dispersion forces can in some cases cause gas hydrates at thermal equilibrium to be coated in a 3-4 $\mathrm{nm}$ thick film of ice, or to contact water directly, depending on which gas they

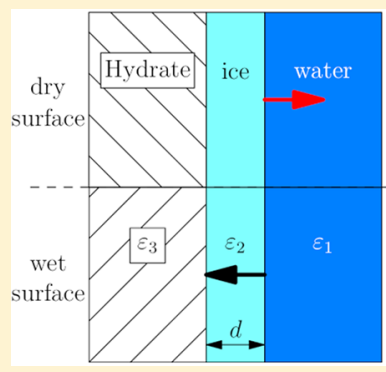
contain. As an example, the results are valid at a quadruple point of the water- $\mathrm{CO}_{2}$ gas hydrate system, where a film is formed not only for the model with pure ice but also in the presence of impurities in water or in the ice layer. These films are shown to significantly alter the properties of the gas hydrate clusters, for example, whether they float or sink. It is also expected to influence gas hydrate growth and gas leakage.

KEYWORDS: Gas hydrates, Interfacial ice formation, Buoyancy, Lifshitz interactions, Dispersion forces

\section{INTRODUCTION}

Gas hydrates are clathrate systems consisting of water and gas molecules forming a solid ice structure. Such systems can naturally be found in ice-cold water; ${ }^{1}$ in particular, they can occur in permafrost, ${ }^{2}$ sediments, ${ }^{3}$ and below the oceans in the seabed. $^{4}$ For the latter, there are particularly interesting examples where gas hydrates are considered important in connection with planetary processes and the implications for life. The aqueous ocean-bearing moons Europa and Enceladus are perhaps the best examples in our solar system beyond Earth where gas hydrates are formed in salty oceans that are favorable for life. ${ }^{5}$ On Mars, methane distribution is associated with subterranean water, implying the presence of methane hydrates. ${ }^{6}$ On Enceladus, giant plumes of erupted gases are observed, and the composition was directly measured to be water, salts, and volatile gases including $\mathrm{CO}_{2}, \mathrm{CO}, \mathrm{N}_{2}, \mathrm{H}_{2} \mathrm{~S}$, and methane. ${ }^{7,8}$ Several hypotheses consider gas hydrates to be important for the creation of volatile enriched plumes and for the composition of ice layers beneath and/or entrained into, or sprayed onto the outer surface of Enceladus. ${ }^{9-11}$ In particular, type II gas hydrates on Enceladus and Europa are calculated to be less dense than water and can float in their respective oceans. They are thereby available for incorporation into the overlying thick ice layer of each icy moon. Type $\mathrm{I}_{\mathrm{CO}_{2}}$ hydrates are at a density where their positive or negative buoyancy is uncertain. ${ }^{9,12-15}$ However, if a layer of water ice forms on these gas hydrates in the presence of ice cold liquid water, then the growth of such hydrate crystals may be limited by the capping effect. This may have an impact on their

Received: January 18, 2019

Revised: March 27, 2019

Accepted: April 9, 2019

Published: April 9, 2019 
buoyancy and thus on the hypothesized composition of the ice layers in Enceladus and Europa, with obvious implications for the composition of their plumes and their potential to sample the underlying oceans and any harbored life.

On Earth, methane hydrates occur naturally and in engineered situations. Large reservoirs of methane hydrates occur in sediments of deep oceans basins, at shallower depths in the sediments of arctic sea shelves, and in deep permafrost regions. In the case of certain hydrates in natural or engineering contexts, these can be stabilized outside their window of thermodynamic stability by formation of an ice layer-this phenomenon is termed "self-preservation" or "anomalous preservation". 16,17 These self-preserving ice layers have been observed, for example, in methane, $\mathrm{CO}_{2}$ and $\mathrm{N}_{2}$ type I hydrates, but not for $\mathrm{H}_{2} \mathrm{~S}$ hydrates. ${ }^{16}$ In all these cases, understanding whether a layer of ice forms on the hydrate has implications for exploration and production of fossil fuels, and also for understanding the potential for methane contribution to greenhouse gases as the planet becomes warmer.

In all of the contexts above, hydrates are usually surrounded by ice cold water. Depending on the gas hydrate structure with respect to contributions and volume fractions, it turns out that some hydrates form an ice interface to water, whereas others do not. The latter have a wet surface. The ones with a gas hydrate-ice interface may be considered to have a dry surface. The prediction of the wet or dry surface cannot be made easily. In the present Article, we address this issue by considering a planar three-layer system, as depicted in Figure 1, namely, a gas

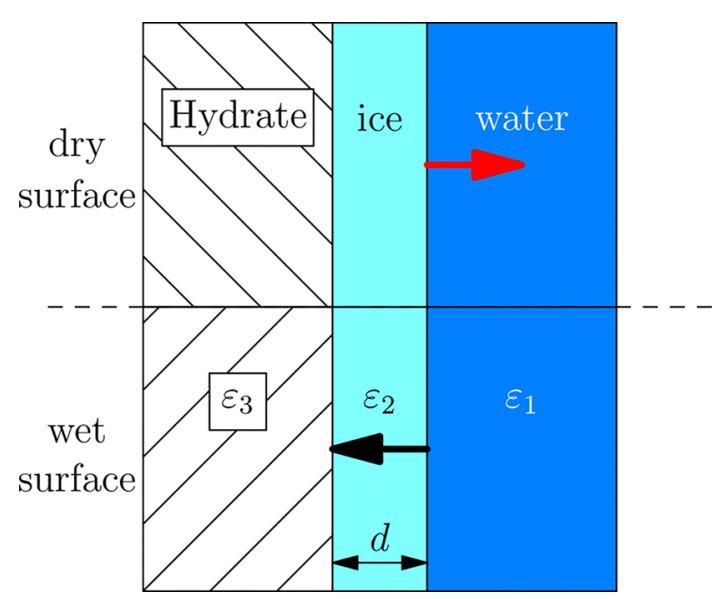

Figure 1. Schematic figure of the considered arrangement. A gas hydrate surface $\left(\varepsilon_{3}\right)$ on the left, separated by an ice layer $\left(\varepsilon_{2}\right)$ of thickness $d$ from a water layer $\left(\varepsilon_{1}\right)$. A dry surface is from a repulsive Casimir force at the ice-water interface, which yields a stable ice interface. In contrast, an attractive force results in a wet surface due to the vanishing of the ice interface.

hydrate layer, an ice sheet, and a water layer. Thus, we assume that initially all hydrates are covered by an ice interface. We estimate the Casimir force acting on the outside at the icewater interface, i.e., the pressure acting on the system. Depending on the sign of the Casimir force, it will work toward growth or melting of the thin ice sheet. We assume that the temperature is at the quadruple point of the water-gas hydrate system, where the liquid water, solid ice, the vapor phase of the gas forming the hydrate, and the solid gas hydrate coexist, which is characterized by the parameters $T=273.1 \mathrm{~K}$ and $p=12.56$ bar in case of $\mathrm{CO}_{2}{ }^{18}$ The corresponding point for $\mathrm{CH}_{4}$ is $T=272.9 \mathrm{~K}, p=25.63 \mathrm{bar}$; for $\mathrm{H}_{2} \mathrm{~S}$, it is $T=272.8$ $\mathrm{K}, p=0.93$ bar; and for $\mathrm{N}_{2}$, it is $T=271.9 \mathrm{~K}, p=143.38$ bar. As observed by Sloan and Koh, while the quadruple-point pressures vary for these gas molecules, the quadruple point temperatures are all approximately $T=273 \mathrm{~K}^{18}$ As discussed in a review by $\operatorname{Dash}^{19}$ it seems Michael Faraday was first to observe that an attractive pressure acting on an ice layer results in a melting of the ice sheet. ${ }^{19,20}$ It will simply vanish. This kind of consideration is not new. In the past, ice melting at the triple point of water with a nanosized film of water under the influence of Lifshitz forces was discussed. ${ }^{21}$ It was found that a thin water film is energetically favorable up to a certain thickness where it has an energy minimum..$^{21-25}$ The inclusion of retardation resulted in incomplete melting, while a nonretarded approximation predicted complete melting for an ice surface at the triple point of water. ${ }^{21}$

Here we apply Lifshitz theory to estimate the energy of the hydrate-ice system as a function of ice thickness and show that for some gas hydrates the ice film is stabilized at a thickness of 3-4 nm, while for other gas hydrates the ice film is unstable, resulting in direct wetting.

\section{MATERIALS AND METHODS}

Our analysis of the stabilization of a thin ice film between hydrate and aqueous phases centers on minimization of the Casimir interaction energy (also known as Lifshitz free energy). Electrostatic energy due to free ions in the aqueous phase and surface charge at the ice-water interface is also present in the total free energy, but this contribution does not vary with ice thickness and therefore does not contribute to stabilization of the ice layer.

2.1. Dispersion Forces between Solid Bodies. The Casimir interaction energy $F(d)$ (Lifshitz free energy) per unit area between material 1 (water) with dielectric function $\varepsilon_{1}$ and material 3 (gas hydrate), $\varepsilon_{3}$, separated by the distance $d$ across medium 2 (ice), $\varepsilon_{2}$, as depicted in Figure 1 can be written at temperature $T$ as $^{26-29}$

$$
F(d)=\sum_{n=0}^{\infty}\left[g^{\mathrm{TM}}\left(\xi_{n}\right)+g^{\mathrm{TE}}\left(\xi_{n}\right)\right]
$$

where $g^{\mathrm{TX}}(\xi)$ (TX $\left.=\mathrm{TM}, \mathrm{TE}\right)$ denotes the trace over the scattering for transverse magnetic (TM) and transverse electric (TE) Green's function. This fundamental solution comes from the vector Helmholtz equation for the electric field. The primed sum denotes that the $n=0$ term is weighted by a factor one-half. At finite temperature these functions are evaluated at the discrete Matsubara frequencies $\xi_{n}=2 \pi n k_{\mathrm{B}} T / \hbar .^{30}$ The systems in this study, as mentioned, are all studied at their corresponding quadruple point. For the considered three-layer system, the traces over the scattering Green's functions, including multiple reflection in the center layer, can be written (in cgs units) as

$$
g^{\mathrm{TX}}\left(\xi_{n}\right)=\frac{1}{\beta} \int \frac{\mathrm{d}^{2} q}{(2 \pi)^{2}} \ln \left\{1-\mathrm{e}^{-2 \gamma_{2} d} r_{12} \mathrm{TX}_{32} \mathrm{TX}_{3}\right\}
$$

with $\beta=1 /\left(k_{\mathrm{B}} T\right)$, and the Fresnel reflection coefficients are

$$
r_{i 2}^{\mathrm{TM}}=\frac{\varepsilon_{i} \gamma_{2}-\varepsilon_{2} \gamma_{i}}{\varepsilon_{i} \gamma_{2}+\varepsilon_{2} \gamma_{i}}
$$

for TM waves and 


$$
r_{i 2}^{\mathrm{TE}}=\frac{\gamma_{2}-\gamma_{i}}{\gamma_{2}+\gamma_{i}}
$$

for TE waves. We have introduced the imaginary part of the transverse wave vector $\gamma_{i}^{2}=q^{2}+\xi^{2} \varepsilon_{i} / c^{2}$. We assume nonmagnetic media.

2.2. Material Modeling. Dielectric functions (at imaginary frequencies) were taken from Elbaum and Schick. ${ }^{21}$ For ice $\left(\varepsilon_{2}\right)$, data from J. Daniels ${ }^{31}$ (ice JD $_{\text {D }}$ ) and from M. Seki et al. ${ }^{32}$ (ice isS $_{\text {) were used. Elbaum and Schick's data }}{ }^{21}$ was used for water $\left(\varepsilon_{1}\right)$. These dielectric functions are for a system at the triple point of water, close to zero degrees Celsius at low pressure. We assume that they are similar at the quadruple points of the systems considered here. The quadruple pressures vary for the gas molecules considered, but the quadruple point temperature all approximates close to $T=273$ $\mathrm{K} .{ }^{18}$ The pressure variation is low for methane, carbon dioxide, and hydrogen sulfide leading to a variation of the dielectric constant by less than $0.05 \%$ compared to the triple point of water. $^{33}$ In contrast, nitrogen shows a larger enhancement of the pressure leading a deviation of the dielectric constant of water around $1 \%$. The dynamical part of the dielectric function has to distribute these changes over the whole frequency axis in order to satisfy the Kramers-Kronig relationship, which means we can assume that the dielectric function is virtually unaffected by them. The finite frequency parts of the dielectric function of water and ice are further known to be much less temperature dependent than the corresponding dielectric constants (at zero frequency). Due to the solid structure of ice, we neglect the changes of its dielectric function with respect to the high pressure. The final results for ice melting $^{21-25,34,35}$ and water freezing ${ }^{36,37}$ are sensitive to the dielectric functions of ice and water since these are extremely similar when the water is in equilibrium with the ice. We show in Figure 2 the dielectric functions for crystalline $\mathrm{CO}_{2}$, water, and ice.

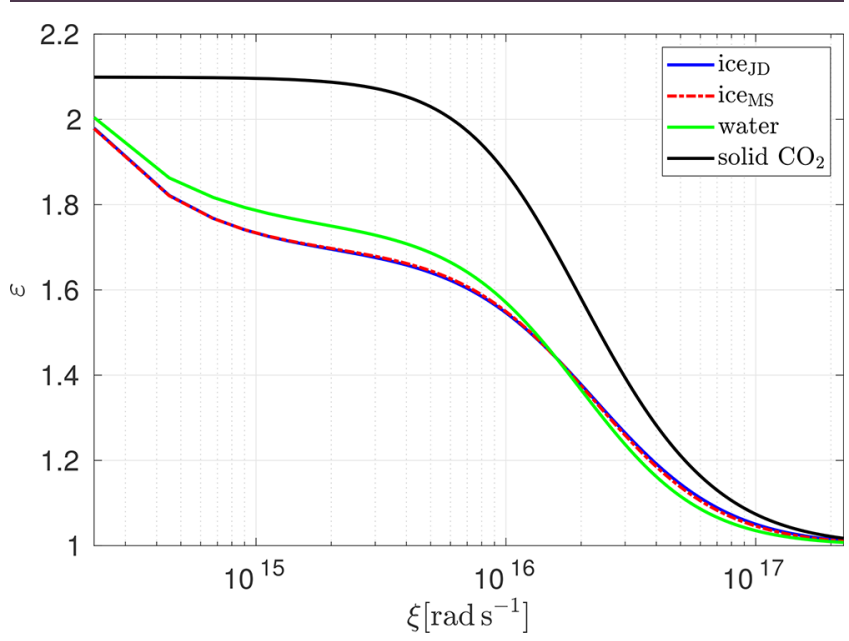

Figure 2. Dielectric functions of crystalline $\mathrm{CO}_{2},{ }^{34}$ both ice models, and water $^{21}$ at $273.16 \mathrm{~K}$.

A model for the dielectric function of a gas hydrate $\left(\varepsilon_{3}\right)$ is derived using the Lorentz-Lorenz model ${ }^{38}$ with the mixing scheme specifically for gas hydrates taken from Bonnefoy et al. $^{39,40}$

$$
\varepsilon_{3}=\frac{1+2 \Gamma}{1-\Gamma}
$$

with

$$
\Gamma=\frac{\varepsilon_{2}-1}{\varepsilon_{2}+2}\left(\frac{n_{\mathrm{wh}}}{n_{\mathrm{i}}}\right)+\frac{4 \pi \alpha_{\mathrm{M}} n_{\mathrm{M}}}{3}
$$

which means that the dominating factors for the dielectric function of gas hydrates are the ice polarizability weighted by density of water in the hydrate relative to pure ice, and the polarizabilities of different gas molecules weighted by their corresponding densities. The mass density of water in pure ice is $0.9167 \mathrm{~g} / \mathrm{cm}^{3}$, ${ }^{33}$ giving the number density of water molecules in pure ice as $n_{\mathrm{i}}=3.06 \times 10^{-2} \AA^{-3}$. The number densities of gas molecules $\left(n_{\mathrm{M}}\right)$ and water molecules $\left(n_{\mathrm{wh}}\right)$ in different gas hydrate structures are tabled in Table 1 with the water/gas number density ratio:

$$
p=\frac{n_{\mathrm{wh}}}{n_{\mathrm{M}}}
$$

Table 1. Hydrate Mass Densities $\left(\rho_{\mathrm{h}}\right)$ and Number Densities of Water $\left(n_{\mathrm{wh}}\right)$ and Gas Molecules $\left(n_{\mathrm{M}}\right)$ in Different Gas Hydrates; the Water/Gas Number Density Ratio Is Denoted $p$

\begin{tabular}{clccc} 
gas molecule & $p$ & $\rho_{\mathrm{h}}\left(\mathrm{g} / \mathrm{cm}^{3}\right)$ & $n_{\mathrm{M}}\left(\AA^{-3}\right)$ & $n_{\text {wh }}\left(\AA^{-3}\right)$ \\
$\mathrm{CO}_{2}{ }^{41}$ & 5.75 & 1.13 & $4.61 \times 10^{-3}$ & $2.65 \times 10^{-2}$ \\
$\mathrm{CO}_{2}{ }^{41}$ & 7.67 & 1.05 & $3.47 \times 10^{-3}$ & $2.66 \times 10^{-2}$ \\
$\mathrm{CO}_{2}{ }^{42}$ & 6.0 & 1.117 & $4.42 \times 10^{-3}$ & $2.65 \times 10^{-2}$ \\
$\mathrm{CH}_{4}{ }^{2}$ & 5.75 & 0.90 & $4.53 \times 10^{-3}$ & $2.60 \times 10^{-2}$ \\
$\mathrm{CH}_{4}{ }^{42}$ & 6.0 & 0.91 & $4.41 \times 10^{-3}$ & $2.65 \times 10^{-2}$ \\
$\mathrm{H}_{2} \mathrm{~S}^{42}$ & 7.0 & 1.044 & $3.92 \times 10^{-3}$ & $2.75 \times 10^{-2}$ \\
$\mathrm{~N}_{2}{ }^{42}$ & 6.0 & 0.995 & $4.4 \times 10^{-3}$ & $2.64 \times 10^{-2}$ \\
\hline
\end{tabular}

Quantum chemical calculations of dynamic polarizabilities at discrete frequencies were represented at arbitrary imaginary frequencies $i \xi$ by fitting to the oscillator model

$$
\alpha_{\mathrm{M}}(i \xi)=\sum_{j} \frac{\alpha_{j}}{1+\left(\xi / \omega_{j}\right)^{2}}
$$

A 5-mode fit has previously been found to describe the dynamic polarizability accurately to a $0.02 \%$ relative error. ${ }^{43}$ The adjusted parameters for a 5-mode model for $\mathrm{CO}_{2}, \mathrm{CH}_{4}$, $\mathrm{N}_{2}$, and $\mathrm{H}_{2} \mathrm{~S}$ are given in our recent work. ${ }^{44}$ Quantum calculations on which the fits were based were taken at a coupled-cluster singles and doubles (CCSD) level of theory ${ }^{45}$ using aug-cc-pVQZ basis sets. ${ }^{46}$

2.3. Product of Reflection Coefficients. As can be observed in eq 2, the Casimir force is determined by the product of reflection coefficients at both interfaces, summed over all frequencies. Thus, the magnitude and sign is given by the balance of areas enclosed by these curves above and below the frequency axis. This behavior is illustrated in Figure 3, where the products of the nonretarded Fresnel coefficients (TM mode) are shown each given by

$$
r_{i 2}=\frac{\varepsilon_{i}-\varepsilon_{2}}{\varepsilon_{i}+\varepsilon_{2}}
$$

One can get insights from this quantity also in cases where retardation matter. Negative values for the product shown in Figure 3 (larger in magnitude for $\mathrm{CO}_{2}$ hydrates than for $\mathrm{CH}_{4}$ 


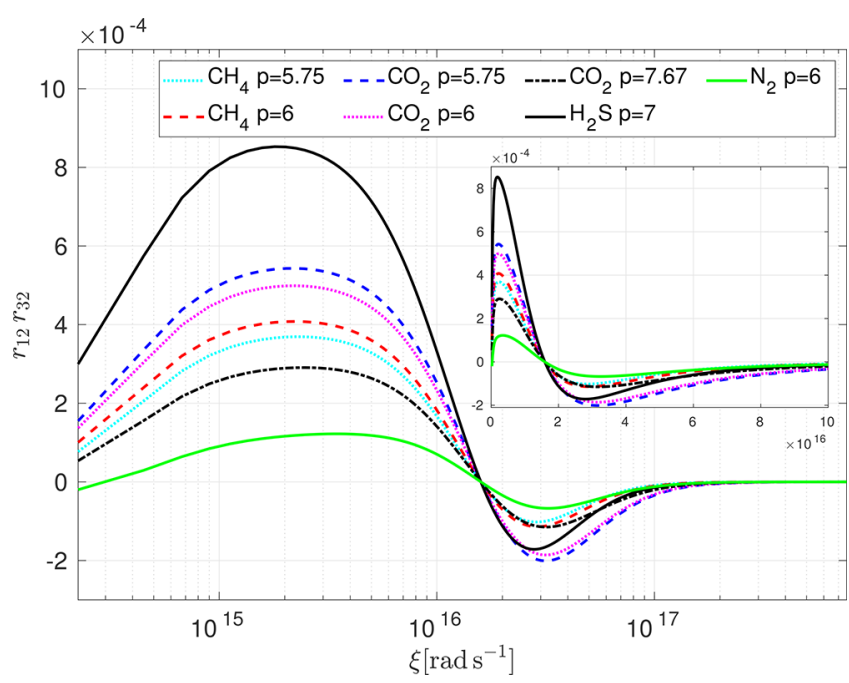

Figure 3. Product of the nonretarded reflection coefficients for the two interfaces. Here, we show these for the three $\mathrm{CO}_{2}$ gas hydrates and the two $\mathrm{CH}_{4}$ gas hydrates that we consider (the inset shows the same on a linear scale).

hydrates) for high frequencies contribute to repulsion. The crossing point at $1.6 \times 10^{16} \mathrm{rad} \mathrm{s}^{-1}$ where $r_{12} r_{32}=0$ corresponds to the frequency where the dielectric function of ice crosses that of pure water, seen in Figure 2. For nonretarded, small film thicknesses, the respective sum over all frequencies (with many more terms for high frequencies than for low frequencies) gives the net sign for the free energy of very thin ice films. Retardation favors the small-frequency contributions and hence screens out high frequency (repulsive) contributions for thicker ice films. It turns out that already for film thicknesses as thin as a few nanometers retardation is important for ice-water related systems. ${ }^{21}$ The net sign in our case is not trivial, and we will demonstrate later that $\mathrm{CO}_{2}$ and $\mathrm{N}_{2}$ hydrates in water behave differently from $\mathrm{CH}_{4}$ and $\mathrm{H}_{2} \mathrm{~S}$ hydrates in water.

2.4. Electrostatic Energy in the Aqueous Phase. Due to dissociation of ice molecules or binding of $\mathrm{H}^{+}$at the icewater interface, that interface is charged, with a negative charge at $\mathrm{pH}$ higher than $3.5 .^{47,48}$ The surface charge of ice facilitates electrostatic physisorption of free ions in the aqueous phase, whether background $\mathrm{H}^{+}$or $\mathrm{OH}^{-}$ions, or salt ions in the case of saline conditions. The associated electrostatic energy and configuration entropy of physisorbed ions forms a significant contribution to the total free energy, which may be evaluated using a Poisson-Boltzmann model to compute the electrostatic potential and ion concentration profiles. ${ }^{48}$ Using such a model, we estimate the electrolyte energy of the aqueous phase (including ice surface charge) to be $-0.23 \mathrm{~mJ} \mathrm{~m}^{-2}$ in pure (without salt) but $\mathrm{CO}_{2}$-saturated water at $\mathrm{pH} 5.8$ in the absence of $\mathrm{Ca}^{2+}\left(\mathrm{CO}_{2}\right.$ saturation means the water phase contains bicarbonate ions at a concentration of $1.58 \mu \mathrm{mol} \mathrm{L} \mathrm{L}^{-1}$ at 1 bar pressure). With salt $(\mathrm{NaCl})$ concentrations of 0.1 or 1 mol L ${ }^{-1}$ added, the electrolyte energy increases in magnitude to -220 and $-272 \mathrm{~m} \mathrm{~m} \mathrm{~m}^{-2}$, respectively. In model seawater ${ }^{49}$ saturated with calcium carbonate at $\mathrm{pH} 8.2$ ( cold seawater $^{50}$ ), the surface charge of ice will be greater, resulting in an electrostatic/electrolyte energy of $-380 \mathrm{~mJ} \mathrm{~m}^{-2}$.

However, it is important to note that in the context of the hydrate-ice-water system, the water-phase electrostatic and free ion adsorption energy is constant for a given $\mathrm{pH}$ and salinity, independent of the thickness of the ice layer or the proximity of the underlying gas hydrate (except, perhaps, in the extreme case of a layer which is only one or two ice molecules thick). That is, this energy does not contribute to the interaction energy considered in this work, which determines the stability of the ice layer.

\section{RESULTS}

3.1. Gas Hydrate Specific Ice Formation. While it is well-known that water can start to freeze from its surface when the temperature goes to zero degrees Celsius, Elbaum and Schick ${ }^{36}$ predicted that dispersion forces do not play a role in this mechanism. In fact, they found that a thin ice film on the surface would have its energy minimum for zero ice film thickness, which would not result in surface freezing on open water surfaces. The underlying mechanism for why ice growth actually occurs at the surface is that large ice structures float with a certain fraction above a water surface due to the lower density of ice. In contrast to their results, we have found that buoyancy combined with dispersion and double layer forces establish an equilibrium where large ice particles float on the surface, while small (micron-sized) ice particles are trapped at a distance below a water surface. ${ }^{48}$ Further, it was shown that ice formation can be induced by dispersion forces near silicawater interfaces (where silica can be used as a model for rock material). ${ }^{37}$

Before presenting the gas hydrates, we first perform calculations for the interaction free energy for an ice film growing on an interface between crystalline $\mathrm{CO}_{2}$ and ice cold water, using the dielectric functions shown in Figure 2. As explained above, the interaction free energy refers to the change in total free energy with respect to ice thickness $d$, after subtracting contributions due to surface charge at the icewater interface and the adsorption of free ions in the water phase (these contributions being constant with respect to d).We see in Figure 4 that this three layer system has an energy minimum corresponding to an equilibrium ice film with thickness $(d)$ between 3.3 and $3.9 \mathrm{~nm}$, depending on the model for the dielectric function of ice. In the remainder of this Article, we use ice ${ }_{J D}$, the Daniels ${ }^{31}$ model for ice, since both

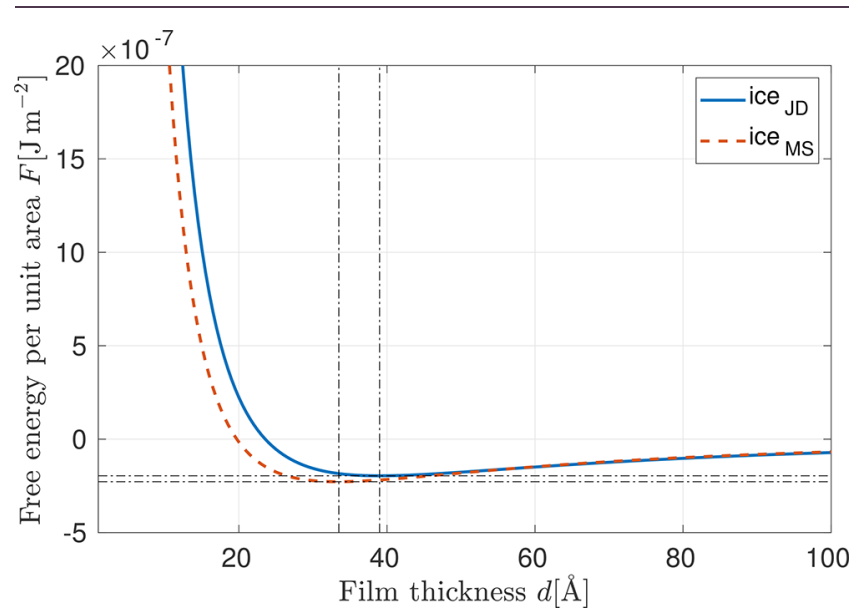

Figure 4. Free energy per unit area (at $273.16 \mathrm{~K}$ ) as a function of ice film thickness on the boundary between the surface of a crystalline $\mathrm{CO}_{2}$ structure and liquid water. It is predicted that at equilibrium an ice film around $d=33 \AA$ for the ice model from Seki et al. and $d=39$ Å for Daniels' model. 
models give very similar results. The thicknesses correlate with the frequency where the dielectric functions of ice and water have a crossing. ${ }^{37}$

Figure 5 shows the free energy as a function of ice film thickness for different gas hydrates in ice cold water. Ice films

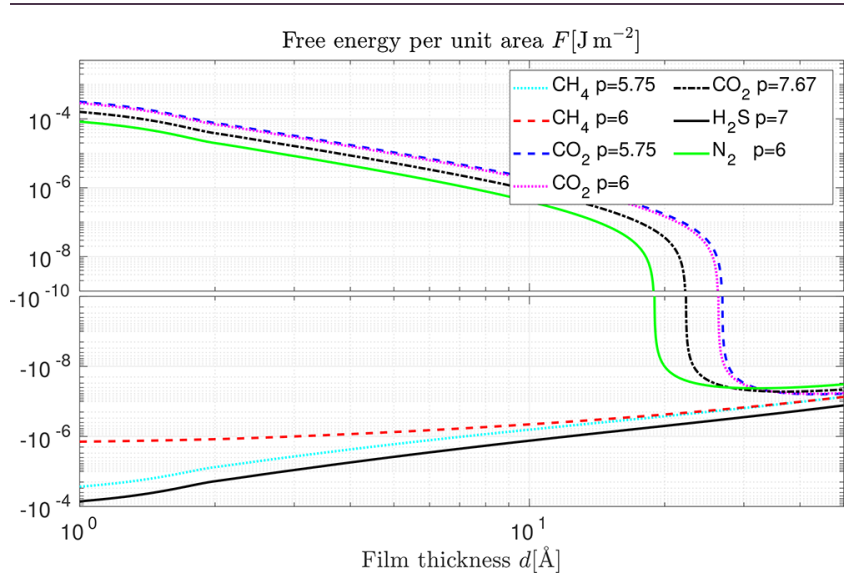

Figure 5. Lifshitz free energy per unit area for a flat three-layer system (water-ice-gas hydrate) as a function of the ice film thickness $d$. For $\mathrm{CO}_{2}$ gas hydrates and the $\mathrm{N}_{2}$ gas hydrate, energy minimum exist corresponding in each case to an equilibrium ice film thickness: $d_{\mathrm{CO}_{2}}=$ 44, 43, and $37 \AA$ for $\mathrm{CO}_{2}$ volume fractions $p=5.75,6$, and 7.67, respectively, and $d_{\mathrm{N}_{2}}=32 \AA$.

are predicted for $\mathrm{CO}_{2}$ hydrates $(d=44,43$, and $37 \AA$ for volume fractions $p=5.75,6$, and 7.67, respectively) and for the $\mathrm{N}_{2}$ hydrate $(d=32 \AA)$ but not for any of the $\mathrm{CH}_{4}$ or $\mathrm{H}_{2} \mathrm{~S}$ hydrates. Due to the deviation of the dielectric function of water ( $1 \%$ for the static value) at higher pressure for the quadruple point of the water-nitrogen system, one finds a deviation of the layer thickness around $\pm 2 \AA$ by assuming an overall deviation of $\pm 1 \%$ over the whole frequency range for its electric susceptibility. This illustrates the maximum error due to the change of the dielectric function.

In the former cases, retardation plays a role at the nanometer scale as it is the reason for the change in the sign of the Lifshitz energy. This model is sensitive to the various dielectric functions that are involved in the system. ${ }^{51}$ While the results are model dependent for the specific combination of materials used, the clear trend is that interfacial ice caps can exist at some gas hydrates in ice cold water, but not for others.

The stark difference in behavior between $\mathrm{CO}_{2}$ or $\mathrm{N}_{2}$ hydrates and $\mathrm{CH}_{4}$ or $\mathrm{H}_{2} \mathrm{~S}$ hydrates can be understood from differences in gas polarizability in the optical/UV spectrum, combined with the difference in the dielectric spectra of water and ice. These are expressed in the maximum and minimum seen in the product of reflection coefficients in Figure 3. The positive value of the product $r_{12} r_{32}$ at low frequencies contributes to stabilization of the water interface toward the hydrate interface, i.e., wetting, with removal of the ice layer. Negative values at high frequencies destabilize the water interface, i.e., stabilize the ice layer. The overall behavior is a balance between these two regimes. As discussed above, the positive and negative regimes ultimately derive from the reflection coefficient $r_{12}$ between liquid water and ice, that is from the crossing in the dielectric functions of ice and cold water at $1.6 \times 10^{16} \mathrm{rad} \mathrm{s}^{-1}$ seen in Figure 2. The effect of the hydrate (via reflection coefficient $r_{32}$ ) is to strengthen or attenuate $r_{12}$. Figure 3 shows that the high frequency stabilization of the ice layer is weaker for $\mathrm{CH}_{4}$ and $\mathrm{N}_{2}$ than for $\mathrm{CO}_{2}$ at all hydrate ratios, while $\mathrm{H}_{2} \mathrm{~S}$ is only weaker than $\mathrm{CO}_{2}$ at higher water/gas ratios. At low frequencies, destabilization of the ice layer is much stronger for $\mathrm{H}_{2} \mathrm{~S}$ than $\mathrm{CO}_{2}$, while weaker for $\mathrm{N}_{2}$. In the balance between low frequency destabilization and high frequency stabilization of the ice layer, high frequencies dominate for $\mathrm{CO}_{2}$, but are insufficiently weak for $\mathrm{CH}_{4}$. In the case of $\mathrm{N}_{2}$, low frequency behavior is weaker than for other gases, so again high frequency stabilization of ice dominates. In the case of $\mathrm{H}_{2} \mathrm{~S}$, low frequency destabilization of the ice layer is stronger than for $\mathrm{CO}_{2}$ and dominates over high frequency stabilization. These patterns follow the underlying polarizabilities of the gas molecules, see Figure 6: the polarizability of $\mathrm{CH}_{4}$ is weaker

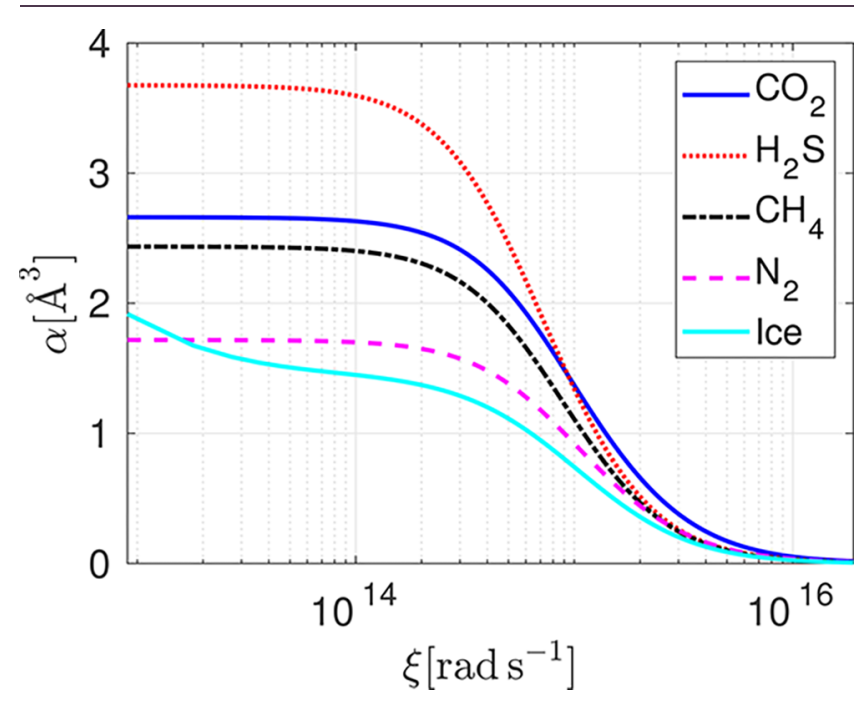

Figure 6. Polarizabilities of gas molecules at imaginary frequencies. The polarizability of a water molecule in ice is also shown for comparison.

than $\mathrm{CO}_{2}$ at all frequencies. The polarizability of $\mathrm{H}_{2} \mathrm{~S}$ is significantly stronger than $\mathrm{CO}_{2}$ at low frequencies, but drops rapidly at high frequencies, crossing $\mathrm{CO}_{2}$ to respond similarly to $\mathrm{CH}_{4}$ in the UV spectrum. The polarizability of $\mathrm{N}_{2}$ is much weaker than other gas molecules, in particular, is much closer to the polarizability of a water molecule. The polarizability per ice molecule is shown in Figure 6 for comparison. This results in an $\mathrm{N}_{2}$ gas hydrate dielectric function closer to that of ice, leading to a smaller reflection coefficient. Stabilization of the ice layer at a hydrate surface is determined predominantly from the polarizability of the gas molecule relative to a water molecule in the optical spectrum around $3 \times 10^{15} \mathrm{rad} \mathrm{s}^{-1}$ (stabilizing water wetting) and in the UV spectrum around $3 \times$ $10^{16} \mathrm{rad} \mathrm{s}^{-1}$ (stabilizing the ice layer).

3.2. Impact of Impurities in Ice and Water on the Stabilized Distance. In the calculations above, we observed a stabilization of the ice layer for $\mathrm{CO}_{2}$ and $\mathrm{N}_{2}$ hydrates caused by the balance of dispersion forces. Ice was taken as pure ice, and it is reasonable to question whether this restriction is suitable. Are these ice layers disrupted by the presence of impurities (molecules of the gases) in the ice and water phases? In order to analyze the behavior of the stabilized region, we apply the Lorentz-Lorenz model ${ }^{38}$ to approximate the dielectric function of a composite $\bar{\varepsilon}_{i}$ of one medium with dielectric function $\varepsilon_{i}$ with embedded gas molecules with dielectric function $\varepsilon_{\mathrm{M}}$ 


$$
\frac{\bar{\varepsilon}_{i}-1}{\bar{\varepsilon}_{i}+2}=p \frac{\varepsilon_{\mathrm{M}}-1}{\varepsilon_{\mathrm{M}}+2}+(1-p) \frac{\varepsilon_{i}-1}{\varepsilon_{i}+2}
$$

with the volume fraction $p$ determining the ratio of gas molecules inside the solid ice phase. Typical concentration of $\mathrm{CO}_{2}$ molecules in water are about $1.4 \times 10^{-5} \mathrm{~mol} / \mathrm{L}^{52,53}$ In the worst case all molecules are equally distributed in the solvent, which leads to an upper bound of the volume fraction of $p=5.6 \times 10^{-7}$ by assuming the largest occupied volume to be $62.28 \AA^{3}$. $^{44}$ Accounting for impurities in ice and water in this way, we estimate their impact on the stabilization distance. The results are depicted in Figure 7 where to illustrate the

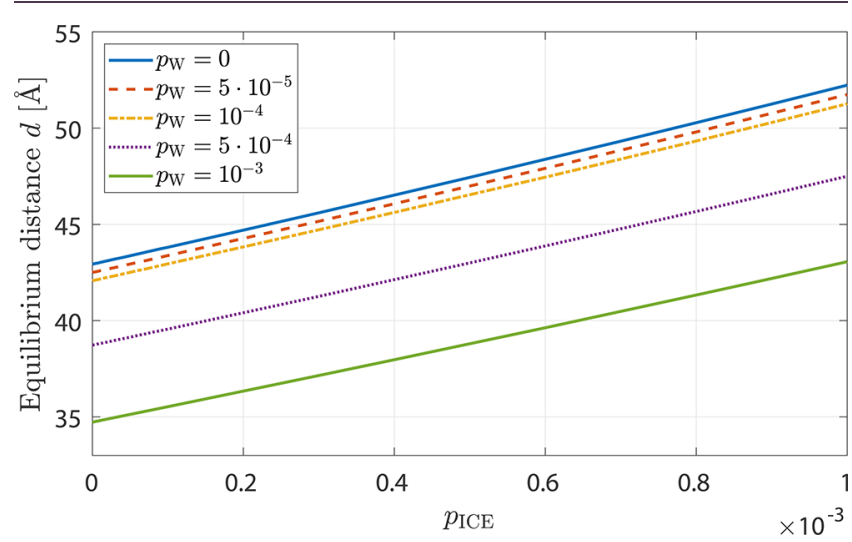

Figure 7. Equilibrium ice film thicknesses (for $\mathrm{CO}_{2}$ gas hydrate with a volume fraction of $p=6$ in equilibrium with water and ice) as a function of volume fraction of impurity $\mathrm{CO}_{2}$ in ice for five different volume fractions of $\mathrm{CO}_{2}$ in water.

point we consider a $\mathrm{CO}_{2}$ gas hydrate with a volume fraction of $p=6$. We observe that for realistic concentrations of $\mathrm{CO}_{2}$ impurities in ice or water the effect on ice sheet thickness can be totally neglected. Using extremely high $\mathrm{CO}_{2}$ concentrations in ice or water enables us to give the result with an upper estimated error due to impurities as $d=43 \pm 10 \AA$ for this system.

Besides the question on the impact of impurities, one can investigate the diffusion of the gas molecules within a gas hydrate layer leading to a dynamical density profile. An extension to address these properties can be performed by the transition to the Casimir force acting in a continuous medium ${ }^{54}$ with the force influenced by the diffusion constant. Such investigations will be part of future studies.

3.3. Size Dependence for Floating of Gas Hydrate Clusters. Buoyancy of gas hydrate particles is of considerable importance for understanding the distribution and composition of ices, water and gases in subglacial water bodies in Antarctica, and on ocean bearing moons of our solar systems and extrasolar planets. Buoyancy of gas hydrates on these water bodies depends on hydrate density and assumed ocean densities.

Lake Vostok, located $4 \mathrm{~km}$ below the Antarctic surface, is an analogue of deeper subglacial oceans of the Jovian and Saturnian moons and is a notable target for astrobiological studies. McKay et al. ${ }^{12}$ suggest the observed lack of gas hydrates accreted at the top of Lake Vostok in Antarctica (density $1.016 \mathrm{~g} \mathrm{~cm}^{-3}$ ) is consistent with formation of relatively dense $\mathrm{CO}_{2}$ hydrates that sink to the lake floor. Mousis et al. ${ }^{14}$ later estimated the densities of type I and II $\mathrm{CO}_{2}$ hydrates in Lake Vostok, concluding that $\mathrm{CO}_{2}$-containing type I hydrates sink above a critical $\mathrm{CO}_{2}$ composition in the lake.

Prieto-Ballesteros et al. ${ }^{13}$ considered buoyancy of type I $\mathrm{CO}_{2}, \mathrm{SO}_{2}, \mathrm{CH}_{4}$, and $\mathrm{H}_{2} \mathrm{~S}$ gas hydrates (space group 223) on Europa, where two extreme models for the density of the ocean water were considered, namely, a eutectic brine of composition $\mathrm{MgSO}_{4}-\mathrm{H}_{2} \mathrm{O}$ with density $1.19 \mathrm{~g} \mathrm{~cm}^{-3}$ and a low salinity water ocean of density $1.0 \mathrm{~g} \mathrm{~cm}^{-3}$. Safi et al. ${ }^{15}$ discuss the buoyancy of $\mathrm{CO}_{2}$ hydrates using two further density estimates of the oceans of Europa (density $1.016 \mathrm{~g} \mathrm{~cm}^{-3}$ ) and Enceladus $\left(1.003 \mathrm{~g} \mathrm{~cm}^{-3}\right)$ and used measured gas hydrate densities. Bouquet et al. ${ }^{9}$ consider the buoyancy of multiple guest clathrates on Enceladus using a calculated high pressure seawater density of $1.030 \mathrm{~g} \mathrm{~cm}^{-3}$, concluding the type I clathrates are marginally denser $\left(1.040 \mathrm{~g} \mathrm{~cm}^{-3}\right)$ than the seawater and type II significantly lighter $\left(0.970 \mathrm{~g} \mathrm{~cm}^{-3}\right)$.

Figure 8 shows the average densities of gas hydrate particles of varying radius, each compositional variant coated in a layer

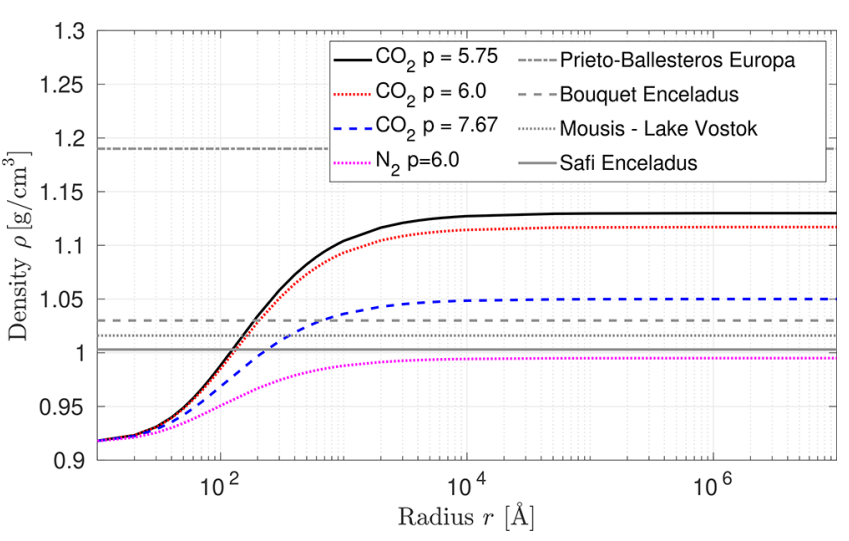

Figure 8. Average densities of gas hydrate particles of varying radius for $\mathrm{CO}_{2}$ hydrates with density fraction $p=5.75$ (solid black line), $p=$ 6.0 (dotted red line), and $p=7.67$ (dashed blue line) and $\mathrm{N}_{2}$ with $p=$ 6.0 (dotted magenta line), each compositional variant coated in a layer of ice of thickness determined by the polarizability and amount of the entrapped gas species as specified above. As comparison we show the water densities in different systems. References for the water/gas number density ratios $p$ are given in Table 1 .

of ice of thickness determined by the polarizability and amount of the entrapped gas species. These were calculated using eq 11 below. The equilibrium ice film thicknesses and densities are given in the text immediately below Figure 5 . The value used for the density of pure ice was $0.9167 \mathrm{~g} \mathrm{~cm}^{-3}$. Horizontal lines represent the estimated or measured density of the ocean/ seawater on the various bodies.

It is quite apparent that for $\mathrm{CO}_{2}$ gas hydrate particles, which are otherwise denser than most models for the water on Enceladus or in Lake Vostok, an equilibrium ice layer of the order of several $\mathrm{nm}$ has a significant impact on the buoyancy of the particles. When the radii of these are below approximately 20-100 nm, the average density drops below values estimated for the ocean water density on Enceladus and consequently will float. We use the following simple expression for the average density of a ice coated gas hydrate cluster (approximated as a sphere)

$$
\rho_{\mathrm{av}}=\frac{\rho_{\mathrm{h}} r_{\mathrm{h}}{ }^{3}+\rho_{\mathrm{i}}\left[\left(r_{\mathrm{h}}+d\right)^{3}-r_{\mathrm{h}}{ }^{3}\right]}{\left(r_{\mathrm{h}}+d\right)^{3}}
$$


Here $\rho_{\text {av }}$ is the average density of mixed particle comprising a gas hydrate core and a shell of water ice, $\rho_{\mathrm{h}}$ densities are given in Table 1 for different gas hydrates, $\rho_{\mathrm{i}}$ is given for ice above, and $r_{\mathrm{h}}$ is the radius of a gas hydrate cluster. Finally, $d$ is the approximate thickness of each ice film at planar water- $\mathrm{CO}_{2}$ gas hydrate and water $-\mathrm{N}_{2}$ gas hydrate interfaces given above. This expression is a macroscopic term, and microscopic properties, such as lattice constants and their mismatch at interface, could be partly included in the mass density of the hydrates and ice.

3.4. Some Further Remarks on Lattice Mismatch of Ice and Hydrates. In general, lattice mismatch at the interface between ice and the gas hydrate impedes the formation of the ice layer. Nevertheless, ice layers on gas hydrates have been observed. ${ }^{16,17,55}$

Indeed, Nguyen et al. ${ }^{55}$ explain the observed formation of ice via a disordered interfacial transition layer between ice and gas hydrates that can relieve the disjoining pressure and aids in explaining nucleation of gas hydrates on ice in the presence of gas and in the formation of ice films on gas hydrates in the well-known, but poorly understood, phenomenon of selfpreservation. Nguyen et al. ${ }^{55}$ state that such a disordered interfacial film must exist at the gas hydrate-ice interface since these interfaces are known to exist during nucleation of gas hydrates from ice-gas mixtures or during self-preservation, despite a lattice matching between the ice and gas hydrate structures. They used molecular dynamics simulations of the gas hydrate-ice interface and found that the interfacial layer between these is always disordered, typically $2-3$ water layers thick, and lowers the surface free energy, which can aid in heterogeneous nucleation of ice from gas hydrates. Such a transitional layer is of negligible effective thickness, and hence, it needs not be explicitly modeled by us and does not effect our results, other than to note that its existence is critical in ours or any model of ice formation on gas hydrates. The observations of nucleation of gas hydrates on ice in the presence of guest gases and the observation of self-preserving films on gas hydrates ${ }^{16,17,55-57}$ provide strong support that our models of gas hydrate-ice systems are physically realistic. Nonetheless, there can also be layered structures with different gas hydrates. We have also considered this case and shown it to have a minor effect on layer thickness (see Figure 7). The lattice mismatch have a minor effect on the density due to the negligible volume of the a transitional interfacial phase that alleviates the lattice mismatch and disjoining pressure. Further, it is well-known that gas diffusion can be dramatically slowed in gas hydrates exhibiting a "self-preservation" or "anomalous preservation" via formation of an ice layer on clathates. Selfpreservation of $\mathrm{CO}_{2}$ gas hydrates by ice layer formation on the gas hydrate has been considered by Falenty and Kuhs. ${ }^{17}$ They state that the relatively low gas permeability observed in "selfpreserved" hydrates is likely explained via annealing of the initial ice film formed on the gas hydrate. This could provide sufficient lifetime for the buoyancy effect that we have demonstrated, rendering the effect persistent over relatively long time scales.

\section{CONCLUSIONS}

In analogy to the premelting layers of ice, ${ }^{21,24,25}$ we found that freezing of gas hydrates in ice cold water is caused by an energy minimum in dispersion energies. This is not expected at water surfaces $^{36}$ but predicted to occur at some water-solid interfaces. ${ }^{37} \mathrm{We}$ find that a significant difference between different gas hydrate surfaces in water lies in whether they are coated with a nanosized interfacial ice cap or not. The result is sensitive to the details in the dielectric functions of the materials involved. However, our results indicate that some hydrates are more likely to have interfaces that are kept dry by an interfacial ice cap. We have seen this trend for three different volume fractions of $\mathrm{CO}_{2}$ hydrates in water as well as for $\mathrm{N}_{2}$ hydrate and crystalline $\mathrm{CO}_{2}$ in water. Other hydrates, $\mathrm{CH}_{4}$ hydrate in water, are more likely to stay wet and have no interfacial ice cap.

A review ${ }^{58}$ a few years ago asked the question if gas hydrate surfaces in air are dry or wet. Our results are consistent with gas hydrate surfaces that are in equilibrium with water molecules in vapor phase. If a film of water is adsorbed on a gas hydrate surface, much thicker than say $10 \mathrm{~nm}$, then our calculations can be extended to predict that a fraction of that water will form an interfacial ice layer between the water film and the surface of the $\mathrm{CO}_{2}$ (or $\mathrm{N}_{2}$ ) hydrates but not so for $\mathrm{CH}_{4}$ (or $\mathrm{H}_{2} \mathrm{~S}$ ) hydrates. These differences for materials, whether their interfaces stay dry or wet, are expected to influence the fluxes of gas molecules into the liquid water and then further toward the surrounding atmosphere. Further, as we discussed above, a dry surface may affect the growth and overall density of gas hydrate crystals. The density of type I $\mathrm{CO}_{2}$ hydrate crystal densities are similar to that predicted for different ice coated ocean waters on Earth, Enceladus, and Europa., ${ }^{9-15}$ The density values in Table 1 for $\mathrm{CO}_{2}$ hydrate suggest a water ice cap layer could make a significant difference in buoyancy when hydrate crystals have diameters in the range of approximately $20-100 \mathrm{~nm}$, based on the 3-4 nm ice films we predict. Indeed, if a layer of interfacial ice cap grows on a hydrate crystal early after nucleation, its growth may be restricted to such small sizes, leading to the formation of nanoscale, ice-capped $\mathrm{CO}_{2}$ hydrate crystals with positive buoyancy.

Besides the requirement of accurate dielectric functions for quantitative predictions of such ice layer thicknesses, the restriction to interactions caused by dispersion forces yields a source of uncertainties. For nonpolar systems, it would be sufficient to neglect electrostatic effects. However, water is a polar medium, thus interactions caused by permanent dipole moments will also play a role and will shift this theory to a more precise one. The extension of the theory of dispersion forces to include permanent dipole moments is of current interest for several groups and will also be part of further investigations. However, a simple estimation of such effects shows a small contribution to the dispersion forces, which is smaller than in the vacuum case due to the shielding effect of the environmental medium.

We have notably shown that the above-mentioned density dependence of the gas hydrates induce a sinking or floating of the particles, which is important for carbon capture and storage via gas hydrates. ${ }^{59}$ The creation of an interfacial ice layer modify the average density of the particle, thus the buoyancy that determines the floating or sinking of the particle. When studying very small gas hydrates, the particle's curvature may be expected to play a role. It can easily be incorporated into theory by changing the geometry from a planar to a spherically layered system. However, since the size of the gas hydrate clusters are much larger than the predicted ice film layer, a planar approximation is expected to give useful estimates. Such investigations will also affect the description of crystallization 
processes in particular for cloud creation ${ }^{60}$ by treating the gas hydrates as analogues of cloud condensation nuclei.

\section{AUTHOR INFORMATION}

\section{Corresponding Authors}

*E-mail: mathias.a.bostrom@ntnu.no.

*E-mail: corkery@kth.se.

*E-mail: d.parsons@murdoch.edu.au.

*E-mail: johannes.fiedler@physik.uni-freiburg.de.

\section{ORCID}

Mathias Boström: 0000-0001-5111-4049

Clas Persson: 0000-0002-9050-5445

Drew F. Parsons: 0000-0002-3956-6031

Johannes Fiedler: 0000-0002-2179-0625

\section{Notes}

The authors declare no competing financial interest.

\section{ACKNOWLEDGMENTS}

We gratefully acknowledge support from the Research Council of Norway (Project 250346), the German Research Council (grant BU 1803/6-1, to S.Y.B. and J.F.; BU 1803/3-1, S.Y.B.), the Research Innovation Fund by the University of Freiburg (to S.Y.B. and J.F.), the Freiburg Institute for Advanced Studies (to S.Y.B.), and FAPERJ (JCNE E-26/203.223/2016). D.F.P. acknowledges the grant of resources from the National Computational Infrastructure (NCI), which is supported by the Australian Government. Data available from authors.

\section{REFERENCES}

(1) Dickens, G. R.; Quinby-Hunt, M. S. Methane hydrate stability in seawater. Geophys. Res. Lett. 1994, 21, 2115-2118.

(2) Max, M. D., Ed. Natural Gas Hydrate in Oceanic and Permafrost Environments; Kluwer Academic Publishers: Washington DC, 2003.

(3) Kwon, T.-H.; Cho, G.-C.; Santamarina, J. C. Gas hydrate dissociation in sediments: Pressure-temperature evolution. Geochem., Geophys., Geosyst. 2008, 9, Q03019.

(4) Mahabadi, N.; Zheng, X.; Jang, J. The effect of hydrate saturation on water retention curves in hydrate-bearing sediments. Geophys. Res. Lett. 2016, 43, 4279-4287.

(5) Nimmo, F.; Pappalardo, R. T. Ocean worlds in the outer solar system. Journal of Geophysical Research: Planets 2016, 121, 13781399.

(6) Fonti, S.; Marzo, G. A. Mapping the methane on Mars. Astron. Astrophys. 2010, 512, 1-6.

(7) Waite, J. H. J.; Combi, M. R.; Ip, W. H.; Cravens, T. E.; McNutt, R. L. J.; Kasprzak, W.; Yelle, R.; J, L.; H, N.; Gell, D.; Magee, B.; Fletcher, G.; G, L.; Tseng, W. L. Cassini ion and neutral mass spectrometer: Enceladus plume composition and structure. Science 2006, 311, 1419-1422.

(8) Postberg, F.; Schmidt, J.; Hillier, J.; Kempf, S.; Srama, R. A saltwater reservoir as the source of a compositionally stratified plume on Enceladus. Nature 2011, 474, 620-622.

(9) Bouquet, A.; Mousis, O.; Waite, J. H.; Picaud, S. Possible evidence for a methane source in Enceladus' ocean. Geophys. Res. Lett. 2015, 42, 1334-1339.

(10) Matson, D. L.; Castillo-Rogez, J. C.; Davies, A. G.; Johnson, T. V. Enceladus: A hypothesis for bringing both heat and chemicals to the surface. Icarus 2012, 221, 53-62.

(11) Matson, D. L.; Davies, A. G.; Johnson, T. V.; Combe, J.-P.; McCord, T. B.; Radebaugh, J.; Singh, S. Enceladus' near-surface $\mathrm{CO}_{2}$ gas pockets and surface frost deposits. Icarus 2018, 302, 18-26.

(12) McKay, C. P.; Hand, K. P.; Doran, P. T.; Andersen, D. T.; Priscu, J. C. Clathrate formation and the fate of noble and biologically useful gases in Lake Vostok, Antarctica. Geophys. Res. Lett. 2003, 30, 35 .
(13) Prieto-Ballesteros, O.; Kargel, J. S.; Fernández-Sampedro, M.; Selsis, F.; Sebastián Martínez, E.; Hogenboom, D. L. Evaluation of the possible presence of clathrate hydrates in Europa's icy shell or seafloor. Icarus 2005, 177, 491-505.

(14) Mousis, O.; Lakhlifi, A.; Picaud, S.; Pasek, M.; Chassefière, E. On the Abundances of Noble and Biologically Relevant Gases in Lake Vostok, Antarctica. Astrobiology 2013, 13, 380-390.

(15) Safi, E.; Thompson, S. P.; Evans, A.; Day, S. J.; Murray, C. A.; Parker, J. E.; Baker, A. R.; M, O. J.; van Loon, J. T. Properties of $\mathrm{CO}_{2}$ clathrate hydrates formed in the presence of $\mathrm{MgSO}_{4}$ solutions with implications for icy moons. Astron. Astrophys. 2017, 600, 1-9.

(16) Takeya, S.; Ripmeester, J. Dissociation Behavior of Clathrate Hydrates to Ice and Dependence on Guest Molecules. Angew. Chem., Int. Ed. 2008, 47, 1276-1279.

(17) Falenty, A.; Kuhs, W. F. Self-Preservation of $\mathrm{CO}_{2}$ Gas HydratesSurface Microstructure and Ice Perfection. J. Phys. Chem. B 2009, 113, 15975-15988. PMID: 19904911.

(18) Sloan, E., Jr.; Koh, C. Clathrate Hydrates of Natural Gases; CRC Press: Boca Raton, FL, 2007.

(19) Dash, J. G. Surface melting. Contemp. Phys. 1989, 30, 89-100.

(20) Landau, L. D.; Lifshitz, E. M. Statistical Physics; ButterworthHeinemann: Oxford, 1980.

(21) Elbaum, M.; Schick, M. Application of the theory of dispersion forces to the surface melting of ice. Phys. Rev. Lett. 1991, 66, 17131716.

(22) Elbaum, M.; Lipson, S.; Dash, J. Optical study of surface melting on ice. J. Cryst. Growth 1993, 129, 491-505.

(23) Wilen, L. A.; Wettlaufer, J. S.; Elbaum, M.; Schick, M. Dispersion-force effects in interfacial premelting of ice. Phys. Rev. B: Condens. Matter Mater. Phys. 1995, 52, 12426-12433.

(24) Dash, J. G.; Fu, H.; Wettlaufer, J. S. The premelting of ice and its environmental consequences. Rep. Prog. Phys. 1995, 58, 115.

(25) Wettlaufer, J. S. Impurity Effects in the Premelting of Ice. Phys. Rev. Lett. 1999, 82, 2516-2519.

(26) Dzyaloshinskii, I.; Lifshitz, E.; Pitaevskii, L. The general theory of van der Waals forces. Adv. Phys. 1961, 10, 165-209.

(27) Parsegian, V. A. Van der Waals forces: A Handbook for Biologists, Chemists, Engineers, and Physicists; Cambridge University Press: New York, 2006.

(28) Ninham, B. W.; Lo Nostro, P. Molecular Forces and Self Assembly in Colloid, Nano Sciences and Biology; Cambridge University Press: Cambridge, 2010.

(29) Buhmann, S. Y. Dispersion Forces I: Macroscopic Quantum Electrodynamics and Ground-State Casimir, Casimir-Polder and van der Waals Forces; Springer: Heidelberg, 2012.

(30) Buhmann, S. Y. Dispersion Forces II: Many-Body Effects, Excited Atoms, Finite Temperature and Quantum Friction; Springer Tracts in Modern Physics; Springer: Heidelberg, 2012.

(31) Daniels, J. Bestimmung der optischen Konstanten von Eis aus Energie - Verlustmessungen von schnellen Elektronen. Opt. Commun. 1971, 3, 240-243.

(32) Seki, M.; Kobayashi, K.; Nakahara, J. Optical Spectra of Hexagonal Ice. J. Phys. Soc. Jpn. 1981, 50, 2643-2648.

(33) Linde, D. R., Ed. CRC Handbook of Chemistry and Physics, 86th ed.; CRC Press: Boca Raton, FL, 2005.

(34) Thiyam, P.; Lima, E. R. A.; Malyi, O. I.; Parsons, D. F.; Buhmann, S. Y.; Persson, C.; Boström, M. Effects of van der Waals forces and salt ions on the growth of water films on ice and the detachment of $\mathrm{CO}_{2}$ bubbles. EPL (Europhysics Letters) 2016, 113, 43002 .

(35) Boström, M.; Malyi, O. I.; Thiyam, P.; Berland, K.; Brevik, I.; Persson, C.; Parsons, D. F. The influence of Lifshitz forces and gas on premelting of ice within porous materials. EPL (Europhysics Letters) 2016, 115, 13001.

(36) Elbaum, M.; Schick, M. On the failure of water to freeze from its surface. J. Phys. I 1991, 1, 1665-1668.

(37) Boström, M.; Malyi, O. I.; Parashar, P.; Shajesh, K. V.; Thiyam, P.; Milton, K. A.; Persson, C.; Parsons, D. F.; Brevik, I. Lifshitz 
interaction can promote ice growth at water-silica interfaces. Phys. Rev. B: Condens. Matter Mater. Phys. 2017, 95, 155422.

(38) Aspnes, D. E. Local field effects and effective medium theory: A microscopic perspective. Am. J. Phys. 1982, 50, 704-709.

(39) Bonnefoy, O.; Gruy, F.; Herri, J.-M. A priori calculation of the refractive index of some simple gas hydrates of structures I and II. Mater. Chem. Phys. 2005, 89, 336-344.

(40) Bonnefoy, O.; Gruy, F.; Herri, J.-M. Van der Waals interactions in systems involving gas hydrates. Fluid Phase Equilib. 2005, 231, 176-187.

(41) Ferdows, M.; Ota, M. Density of $\mathrm{CO}_{2}$ Hydrate by Monte Carlo Simulation. Proc. Inst. Mech. Eng., Part C 2006, 220, 691-696.

(42) Makogon, Y. Natural gas hydrates - A promising source of energy. J. Nat. Gas Sci. Eng. 2010, 2, 49-59.

(43) Parsons, D.; Ninham, B. W. Importance of Accurate Dynamic Polarizabilities for the Ionic Dispersion Interactions of Alkali Halides. Langmuir 2010, 26, 1816-1823.

(44) Fiedler, J.; Thiyam, P.; Kurumbail, A.; Burger, F. A.; Walter, M.; Persson, C.; Brevik, I.; Parsons, D. F.; Boström, M.; Buhmann, S. Y. Effective Polarizability Models. J. Phys. Chem. A 2017, 121, 97429751. PMID: 29185741.

(45) Hampel, C.; Peterson, K. A.; Werner, H.-J. A comparison of the efficiency and accuracy of the quadratic configuration interaction (QCISD), coupled cluster (CCSD), and Brueckner coupled cluster (BCCD) methods. Chem. Phys. Lett. 1992, 190, 1-12.

(46) Woon, D. E.; Dunning, J. Gaussian basis sets for use in correlated molecular calculations. III. The atoms aluminum through argon. J. Chem. Phys. 1993, 98, 1358-1371.

(47) Kallay, N.; Čop, A.; Chibowski, E.; Holysz, L. Reversible charging of the ice-water interface: II. Estimation of equilibrium parameters. J. Colloid Interface Sci. 2003, 259, 89-96.

(48) Thiyam, P.; Fiedler, J.; Buhmann, S. Y.; Persson, C.; Brevik, I.; Boström, M.; Parsons, D. F. Ice Particles Sink below the Water Surface Due to a Balance of Salt, van der Waals, and Buoyancy Forces. J. Phys. Chem. C 2018, 122, 15311-15317.

(49) Millero, F. J.; Feistel, R.; Wright, D. G.; McDougall, T. J. The composition of Standard Seawater and the definition of the Reference-Composition Salinity Scale. Deep Sea Res., Part I 2008, $55,50-72$.

(50) Millero, F. J. Thermodynamics of the carbon dioxide system in the oceans. Geochim. Cosmochim. Acta 1995, 59, 661-677.

(51) van Zwol, P. J.; Palasantzas, G.; De Hosson, J. T. M. Influence of dielectric properties on van der Waals/Casimir forces in solidliquid systems. Phys. Rev. B: Condens. Matter Mater. Phys. 2009, 79, 195428.

(52) Stumm, W.; Morgan, J. Aquatic Chemistry: an Introduction Emphasizing Chemical Equilibria in Natural Waters, 1st ed.; John Wiley \& Sons: New York, 1981.

(53) Schulz, K.; Riebesell, U.; Rost, B.; Thoms, S.; Zeebe, R. Determination of the rate constants for the carbon dioxide to bicarbonate inter-conversion in $\mathrm{pH}$-buffered seawater systems. Mar. Chem. 2006, 100, 53-65.

(54) Parashar, P.; Milton, K. A.; Li, Y.; Day, H.; Guo, X.; Fulling, S. A.; Cavero-Peláez, I. Quantum electromagnetic stress tensor in an inhomogeneous medium. Phys. Rev. D: Part. Fields 2018, 97, 125009.

(55) Nguyen, A. H.; Koc, M. A.; Shepherd, T. D.; Molinero, V. Structure of the Ice-Clathrate Interface. J. Phys. Chem. C 2015, 119, 4104-4117.

(56) Henley, H.; Thomas, E.; Lucia, A. Density and phase equilibrium for ice and structure I hydrates using the GibbsHelmholtz constrained equation of state. Chem. Eng. Res. Des. 2014, 92, 2977-2991. Advances in Thermodynamics for Chemical Process and Product Design.

(57) Khurana, M.; Yin, Z.; Linga, P. A Review of Clathrate Hydrate Nucleation. ACS Sustainable Chem. Eng. 2017, 5, 11176-11203.

(58) Maeda, N. Is the Surface of Gas Hydrates Dry? Energies 2015, $8,5361-5369$.

(59) Hölz, S.; Swidinsky, A.; Sommer, M.; Jegen, M.; Bialas, J. The use of rotational invariants for the interpretation of marine CSEM data with a case study from the North Alex mud volcano, West Nile Delta. Geophys. J. Int. 2015, 201, 224-245.

(60) Khain, A. P.; BenMoshe, N.; Pokrovsky, A. Factors Determining the Impact of Aerosols on Surface Precipitation from Clouds: An Attempt at Classification. J. Atmos. Sci. 2008, 65, 17211748. 